\title{
Management of non-reducing disc derangement in a young patient using progressive muscle relaxation technique
}

\begin{abstract}
Internal derangements of the temporomandibular joint are conditions in which the articular disc has become displaced from its original position over the condylar head. Pain, stress, functional and social handicap are the common reasons for which the patients seek treatment. Various modalities for management include medication, physiotherapy, occlusal splints, self-management strategies, and interventions based on cognitive behavioral approaches. At present, conservative means are preferred to surgical modalities as they are less-aggressive, economical and have comparable successful outcomes. Progressive Muscle Relaxation adapted from Edmund Jacobson (1938) is easy to learn and has been advocated to manage dental anxiety in the past. This article presents a unique case report where this novel technique has been used to manage a young patient with articular disc derangement with a 6-month follow-up showing successful functional and psycho-social rehabilitation of the young patient.
\end{abstract}

Keywords: pain, stress, articular disc derangement, progressive muscle relaxation, novel technique
Volume I 3 Issue 4 - 2020

\author{
Rishi Tyagi, Namita Kalra,Amit Khatri, Puja \\ Sabherwal, Padma Yangdol \\ Department of Pedodontics and Preventive Dentistry, \\ University College of Medical Sciences, India
}

Correspondence: Puja Sabherwal, University College of Medical Sciences, GTB Hospital, Delhi-I I0095, India, Tel99998595।3,Email sabherwalpuja@gmail.com

Received: May 01, 2019 | Published: August 10, 2020
Abbreviations: TMDs, temporomandibular disorders; PD, panic disorder; GAD, generalized anxiety disorder; GCPS, graded chronic pain scale; MRI, magnetic resonance imaging; PHQ, patient health questionnaire; PMR, progressive muscle relaxation

\section{Introduction}

"Temporomandibular disorders" (TMDs) is a collective term for conditions that involve pain and/or dysfunction of the temporomandibular joint (TMJ), and the related structures". The degenerative changes in the TMJ are due to the inability of the TMJ to withstand the dynamic forces with the help of flexibility of its specific structure, tissue repair and functional remodeling. ${ }^{2}$ The development of revised RDC/TMD Axis I, II diagnostic algorithms were subsequently demonstrated to be valid for the most common temporomandibular joint disorders. The RDC/TMD are based on a biobehavioral model of pain, including two main axes: physical signs and symptoms (axis I) and psychological and disability factors (axis II). ${ }^{3}$

The Revised RCD/ TMD suggested a 'Diagnostic Criteria for the Most Common Intra-articular Temporomandibular Disorders' as follows- 1. Disc Displacement with Reduction 2. Disc Displacement with Reduction with intermittent locking 3. Disc Displacement without reduction with limited mouth opening 4. Disc Displacement without reduction without limited mouth opening 5. Degenerative joint disease 6. Subluxation ${ }^{1,3}$.

Conservative treatments for TMD include medication, occlusal splints, physiotherapy, self-management strategies, and interventions based on cognitive behavioral approaches. ${ }^{4,5}$ At present, conservative treatment is preferred over surgery as two have comparable treatment outcomes. Relaxation therapy involves self-regulation techniques to reduce stress, pain and muscle tension. ${ }^{6}$

Progressive Muscle Relaxation adapted from Edmund Jacobson (1938) is easy to learn and has been advocated to manage dental anxiety. ${ }^{7}$ The process of Progressive Muscle Relaxation is based on the basic principle of muscle physiology, that when a muscle is tensed, releasing the tension will lead to relaxation in the muscle? Muscle Relaxation Techniques have been found to be effective and have been used successfully in the past for providing relief in panic disorder (PD) and generalized anxiety disorder (GAD). ${ }^{8}$ Relaxation techniques have been advocated as a strategy for the management of Temporomandibular Disorders and could have a positive influence on maximal mouth opening and pain, but there is limited evidence for the same. ${ }^{9}$

This case report demonstrates this less conventionally used strategy i.e. Progressive Muscle Relaxation Therapy to conservatively manage Non-Reducing Disc Derangement in a young female patient with limited mouth opening.

\section{Case report}

A 15-year-old female patient reported to the Department of Pedodontics and Preventive Dentistry with the chief complaint of pain in front of right ear region and difficulty in eating since 6 months. A detailed case history record was prepared. The patient reported episodes of pain of moderate intensity in the right pre-auricular region since 6months which exacerbated during wide mouth opening and lateral and protrusive movements of the mandible. The pain was relieved slightly with medication. Pain was found to be referred to the jawline and ear region. No headaches were reported. The temporal region, temporalis and trapezius muscles were non-tender on palpation. The masseter and sternocleidomastoid muscles were tender on palpation. Clicking sounds were present during opening movements of the jaw (Figures $1 \mathrm{~A}-1 \mathrm{G})$. Associated reduction in mouth opening was present with vertical incisor overlap of $27 \mathrm{~mm}$ (Figure $2 \mathrm{~A}$ ).

Based on the above-mentioned history and clinical examination a diagnosis of Disc Displacement without reduction with limited mouth opening associated with local myalgia was made (Axis I) [ICD-9 524.63; ICD-10 M26.63]. ${ }^{3}$ To establish a baseline for Axis II (Psychosocial parameters) the Patient Health Questionnaire-4 (PHQ- 
4) and the Graded Chronic Pain Scale (GCPS) were administered. The PHQ-4 revealed a positive score ( $>3$ ) for the Anxiety and Depression subscales with a total score of 6 on each subscale. The GCPS revealed Characteristic Pain Intensity score of 3 points, Disability Score of 3 points and Disability Point score of 3 points. This was classified as Grade III i.e. High Disability and moderately limiting with a Disability point score between 3-4. (Regardless of Characteristic Pain Intensity).

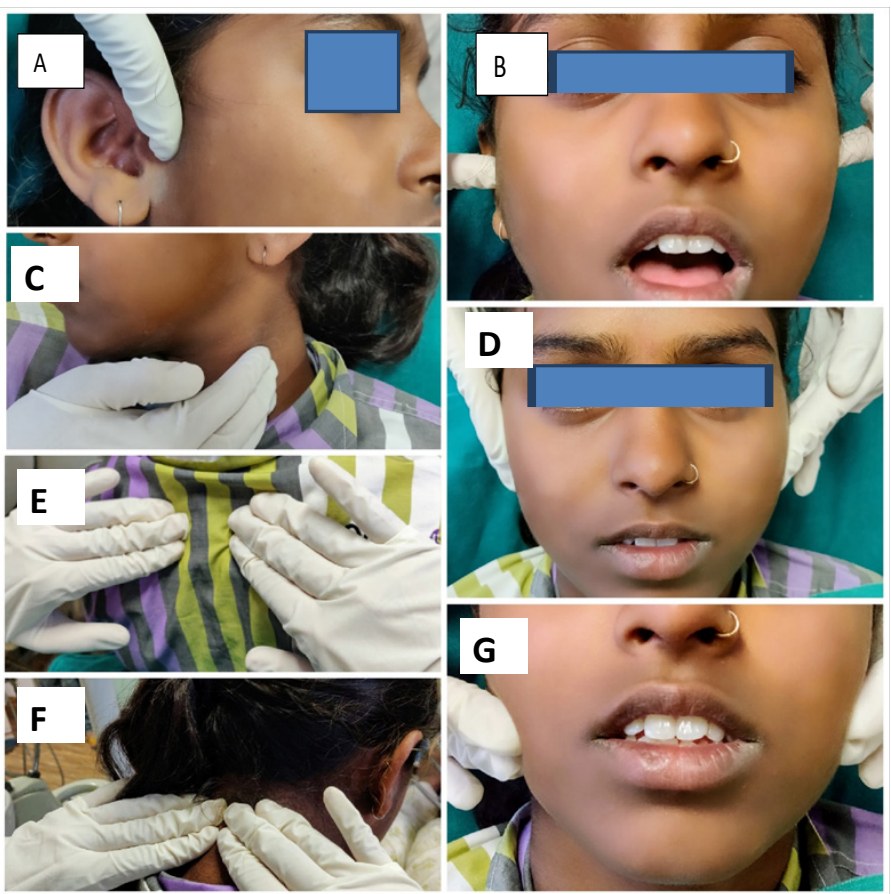

Figure I A Palpation of the lateral pole of Temporomandibular joint; B-Palpation of the joints during mouth opening and closing; C- Palpation of the sternocleidomastoid; D- Palpation of the Temporalis muscle; E,F-Palpation of the Trapezius muscle; G- Palpation of the Masseter muscle.

A conservative management regime was started for the patient i.e. to avoid wide mouth opening, soft diet, and warm moist fermentation of the region.

The patient was then trained in Progressive Muscle Relaxation by a post-graduate resident in Pediatric Dentistry who had been trained in this technique. The patient was asked to lie down in supine position, close her eyes and inhale deeply from the nose and exhale deeply from the mouth (5-10times). The patient was then told to shift awareness to the muscles of the face and slowly relax the jaw, shoulders and observe the rise and fall of the tummy. Relaxation instructions were then read to the patient using voice modulation and maintaining a calm environment as per the script (Figure 3). ${ }^{10,11}$ At the end of the instructions the patient was asked to slowly move finger tips, toes and neck and get up with the aid of support.

This initial training was carried out over duration of 30 minutes for 3days consecutively. The patient was then asked to perform this Relaxation as trained, at home for 30minutes each day on an empty stomach. She was recalled for a follow up after 2 weeks. At the end of 2 weeks she reported marked reduction in the pain and distress i.e. Pain subscale score dropped from 6 to 3 and distress subscale score reduced from 6 to 4 as per the PHQ-4. The mouth opening improved with vertical incisor overlap of $31 \mathrm{~mm}$. However, she still reported pain during wide mouth opening, lateral and protrusive movements of the mandible. The training for the Progressive Muscle Relaxation technique was reinforced. The patient was asked to perform the Relaxation at home and followed up after 4weeks, 8weeks, 12weeks and 24weeks. At the end of 24weeks she reported a score of 1 on the Pain subscale and 2 on the depression subscale as per the PHQ-4. The GCPS scores were as follows Characteristic Pain Intensity score of 0 points, Disability score of 0 points and Disability Point score of 0 points.

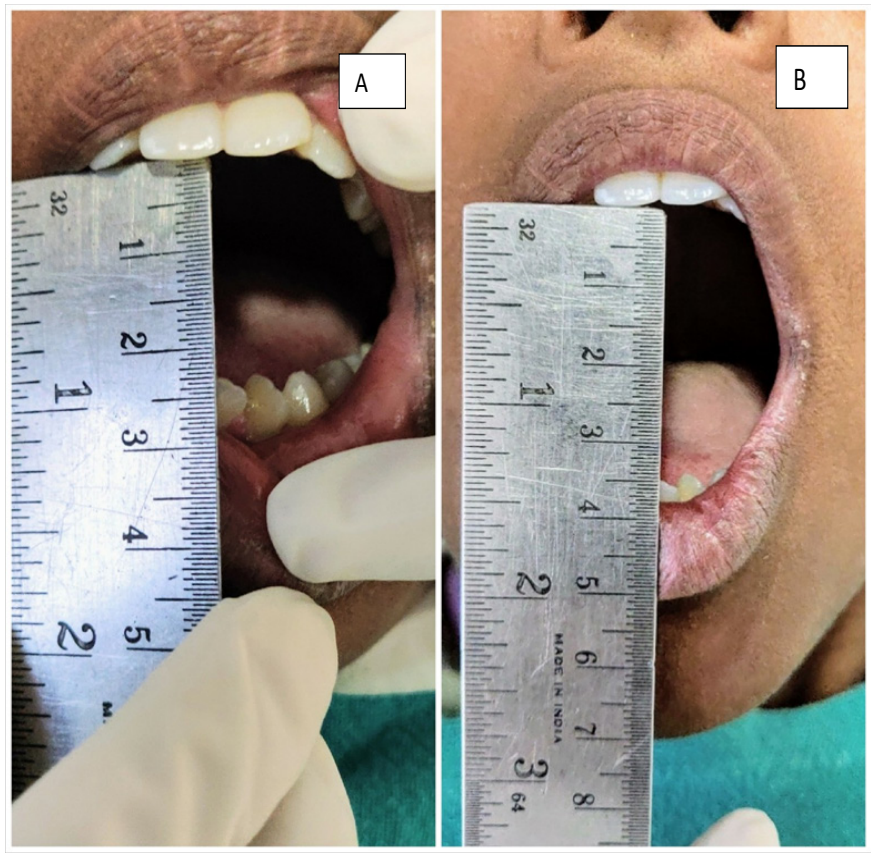

Figure 2 A- Pre-operative mouth opening; B-Mouth opening post 6months.

The vertical incisor overlap was $38 \mathrm{~mm}$ and patient was able to eat food easily. Her overall perspective had improved as her mood was more cheerful during the day and productivity had improved at school and housework. She was able to attend social events with friends and family.

\section{Discussion}

A temporomandibular disorder (TMD) is a common disorder affecting up to $33 \%$ of individuals during their lifetime. TMD is perceived frequently as a repetitive motion disorder of the masticatory structures and is similar to other musculoskeletal disorders in the body. ${ }^{12}$

Disc displacement without reduction with limited opening is a capsular disorder of the condyle and articular disc complex. It manifests as pain and persistent limited mandibular opening that does not reduce with patient or dental surgeon performing a manipulative maneuver. Examination reveals limited opening less than $40 \mathrm{~mm}$ between maxillary and mandibular incisal edges with opening assisted by the dentist. Imaging techniques like the Magnetic Resonance Imaging (MRI) may be used to confirm diagnosis. ${ }^{3}$ In general, management of TMJ-related conditions becomes necessary when dysfunction or pain are present. ${ }^{12}$ In the past, several cases were treated conservatively using thermotherapy, physical therapy, 
interocclusal splints, cryotherapy and drugs, and the ones which failed to reach desired result were referred to surgery and subjected to more aggressive procedures, such as disc anchorage and/or TMJ arthroplasty. ${ }^{13}$ The self-limiting nature of TMDs and the high efficacy of low-cost conservative care indicate that such treatment should be the initial treatment of choice. ${ }^{14}$ Only after failed attempts at conservative care should more aggressive methods be considered.

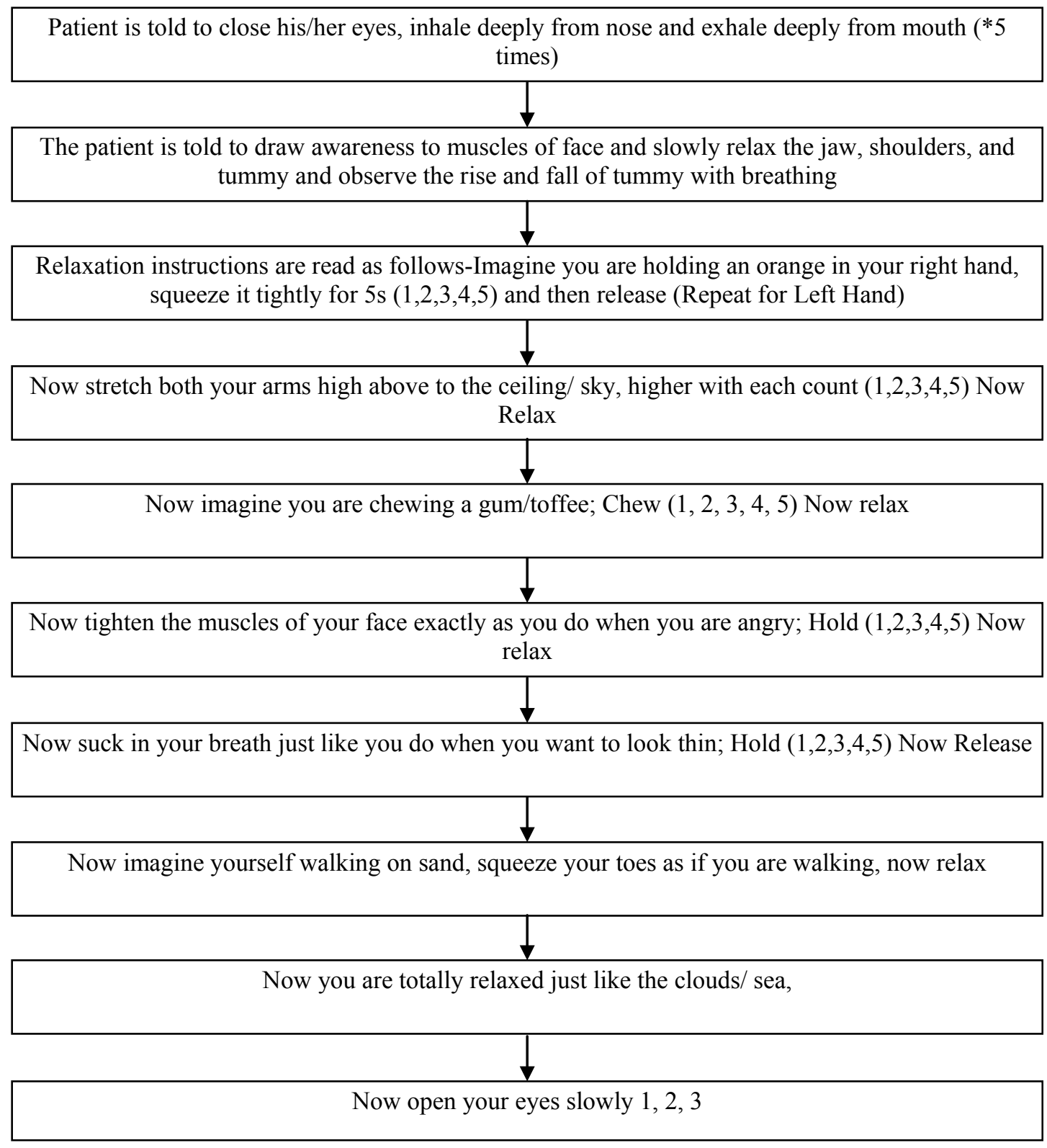

Figure 3 Progressive muscle relaxations.

Keeping in mind that conservative techniques play a pivotal role in the efficient management of Temporomandibular disorders - the case presents therapeutic management in a young female patient using the Progressive Muscle Relaxation technique adopted from Jacobson (1938).

Relaxation therapies like Jacobson's technique involve selfregulation techniques aimed at reducing pain-induced stress and muscle tension. These techniques could have a positive influence on pain intensity and maximal mouth opening, but there is lack of evidence to prove the same. ${ }^{9}$

Nonetheless, beneficial effects of Hypnosis/Relaxation on the maximum mouth opening and maximal pain experienced have been suggested by Zhang et al. ${ }^{15}$ In the presented case, the primary therapeutic goal was to eliminate the pain and distress, and improve mandibular function for the patient. The patient's condition was found to improve with results evident as early as 2 weeks. The patient 
remained asymptomatic at the end of 6 months follow-up. Although, the use of Muscle Relaxation techniques has been advocated in the management of Temporomandibular disorders, its use in routine practice requires further research and need for standardization. ${ }^{6}$ The procedure used in Progressive muscle relaxation is relatively simple but will require an investment of time, firstly to teach the patient and then for the patient to practice at home in order to master the technique. Thus, the compliance of the patient plays a crucial role for the successful management of the condition.

\section{Conclusion}

To summarize, the efficacy of simple conservative management techniques in the management of Temporomandibular Disorders should not be undermined. Progressive Muscle Relaxation ${ }^{10,11}$ is a promising technique for use with children and adolescents which may benefit pain, distress and limited mandibular function associated with Temporomandibular disorders. To bring this technique into routine practice more research, standardization and operator training is the need of the hour.

\section{Acknowledgments}

None.

\section{Conflicts of interest}

Author declares that there are no conflicts of interest.

\section{Funding}

None.

\section{References}

1. Young AL. Internal derangements of the temporomandibular joint A review of the anatomy, diagnosis, and management. $J$ Indian Prosthodont Soc. 2015;15(1):2-7.

2. Vrbanović E, Alajbeg IZ. A Young Patient with Temporomandibular Joint Osteoarthritis: Case Report. Acta Stomatol Croat. 2017;51(3):232-239.

3. Schiffman E, Ohrbach R, Truelove E, et al. International RDC/TMD Consortium Network, International association for Dental Research Orofacial Pain Special Interest Group, International Association for the Study of Pain. Diagnostic Criteria for Temporomandibular Disorders (DC/TMD) for Clinical and Research Applications: recommendations of the International RDC/TMD Consortium Network and Orofacia Pain Special Interest Group. J Oral Facial Pain Headache. 2014;28(1):6-27.
4. Ingawalé S, Goswami T. Temporomandibular joint: disorders, treatments, and biomechanics. Ann Biomed Eng. 2009;37(5):976-996.

5. Medlicott MS, Harris SR. A systematic review of the effectiveness of exercise, manual therapy, electrotherapy, relaxation training, and biofeedback in the management of temporomandibular disorder. Phys Ther. 2006;86(7):955-973.

6. Gil-Martínez A, Paris-Alemany A, López-de-Uralde-Villanueva I, et al. Management of pain in patients with temporomandibular disorder (TMD): challenges and solutions. J Pain Res. 2018;11:571-587.

7. Armfield JM, Heaton LJ. Management of fear and anxiety in the dental clinic: A review. Aust Dent J. 2013;58(4):390-407.

8. Conrad A, Roth WT. Muscle relaxation therapy for anxiety disorders: it works but how? J Anxiety Disord. 2007;21(3):243-624.

9. Aggarwal VR, Lovell K, Peters S, et al. Psychosocial interventions for the management of chronic orofacial pain. Cochrane Database Syst Rev. 2011;(11):CD008456.

10. Karasu TB, Karasu SR. Psychoanalysis and psychoanalytic psychotherapy. In: Sadock BJ, Sadock VA, Ruiz P, editors. Kaplan \& Sadock's Comprehensive Textbook of Psychiatry. 9th edn. Philadelphia: Lippincott Williams \& Wilkins; 2009. p. 2787-2788.

11. Children's Anxiety Institute. How to Teach Children Progressive Muscle Relaxation. 2019.

12. Wright EF, North SL. Management and treatment of temporomandibular disorders: a clinical perspective. J Man Manip Ther. 2009;17(4):247254.

13. Vasconcelos BEV, Bessa-Nogueira RV, Rocha NS. Artrocentesis of temporomandibular joint: evaluation of the results and review of literature. Rev Bras Otorrinolaringol. 2006;72(5):634-638.

14. Garefis P, Grigoriadou E, Zarifi A, et al. Effectiveness of conservative treatment for craniomandibular disorders: a 2-year longitudinal study. J Orofac Pain. 1994;8(3):309-314.

15. Zhang Y, Montoya L, Ebrahim S, et al. Hypnosis/relaxation therapy for temporomandibular disorders: a systematic review and metaanalysis of randomized controlled trials. J Oral Facial Pain Headache. 2015;29(2):115-125. 